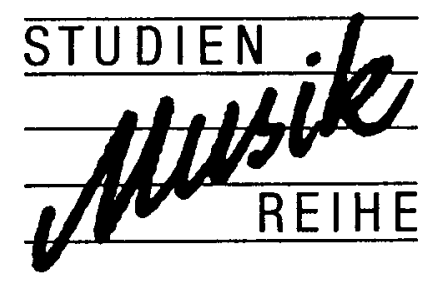

Herausgegeben von

Sabine Schutte und

Franz Niermann 
Sabine Schutte

\section{Die Musik kommt!}

Zur politischen und sozialen Funktion von Marsch- und Tanzmusik

J. B. Metzlersche Verlagsbuchhandlung Stuttgart 


\section{Die Musik kommt!}

Zur politischen und sozialen Funktion von

Marsch und Tanzmusik

Herausgegeben von Sabine Schutte und Franz Niermann

Als Begleitmaterial ist eine Musikkassette (Best.-Nr. 70211) erhältlich.

Die Hörbeispiele im Buch sind mit (H1) etc. gekennzeichnet.

Der Titel dieses Unterrichtswerkes - Die Musik kommt! - entspricht dem auf den Seiten $19 f f$. abgedruckten Musikstück von Oscar STRAus nach dem gleichnamigen Gedicht von Detlev von Liliencron.

Wir danken dem Richard Birnbach Musikverlag (Lochham) für die Reprovorlage des Titelblatts einer Notenausgabe aus dem Jahre 1901.

Eine für Klavierspieler besonders geeignete Fassung zu »Die Musik kommt « ist bei diesem Verlag erhältlich.

ISBN 978-3-476-20339-7

ISBN 978-3-476-99362-5 (eBook)

DOI 10.1007/978-3-476-99362-5

Alle Rechte vorbehalten

(C) 1988 Springer-Verlag GmbH Deutschland

Ursprünglich erschienen bei J. B. Metzlersche Verlagsbuchhandlung

und Carl Ernst Poeschel Verlag GmbH in Stuttgart 1988 


\section{Vorwort der Herausgeber}

Die vorliegenden Unterrichtsreihen gehören in den Rahmen des Generalthemas 'Musik und Politik und sind dazu gedacht, das Moment des Politischen in der Musik historisch zu konkretisieren. Der Zusammenhang von Musik und Politik kann nicht abstrakt, sondern nur in einer bestimmten historischen Situation erkennbar werden. Der hier gewählte zeitliche Rahmen umfaßt die Zeit des deutschen Kaiserreichs und das erste Jahrzehnt der Weimarer Republik, die sogenannten 'goldenen $z$ wanziger Jahre.

\section{Marschmusik und Gassenhauer}

In den Kapiteln 1-6 geht es vorwiegend um Marschmusik in unterschiedlichsten Formen, die in der Kaiserzeit eine bestimmte Rolle hatte. Ihre zwiespältige Funktion steht dabei im Mittelpunkt der Fragestellungen. Einerseits trägt diese Musik dazu bei, viele für die herrschendeStaatsidee nützliche Gefühls- und Verhaltensmuster in der psychischen Struktur der Deutschen zu verankern. Die preußische Disziplin, die sich im Marsch ausdrückt, gerät andererseits aber ins Wanken, wenn der Marsch als belebendes Element in Operetten und Possen erscheint oder durch Hinzufügung parodistischer Texte zum `Gassenhauer und Schlager umfunktioniert wird. Und wenn dazu auch noch getanzt wird, dann ändert sich die Bedeutung der Musik wiederum: Das Publikum hatte wohl kaum das preußische Militär im Kopf, wenn es zu den bekannten Marschrhythmen von WALTER KoLLo einen flotten sSchieber aufs Parkett legte. Oder?

\section{Tanzmusik und Jazz}

Die Kapitel 7-9 sind der Tanzmusik von damals gewidmet. Im Vordergrund stehen die Tänze, die mit dem Marsch verwandt sind. Eine Ausnahme bildet Kapitel 7 mit seinen Ausführungen über den Walzer, der als Inbegriff der salten Tänze des 19. Jahrhunderts und Gegensatz zu den marschverwandten Schreittänzen wie `One- und Twostep`, 'Ragtime،, 'Cakewalk ‘ oder 'Foxtrott ‘ dargestellt ist. Bei der Tanzmusik wurdeder Schwerpunkt auf die Fragestellung gelegt, weshalb die neuen, damals als 'Jazz، bezeichneten Tanzschlager eine solche Faszination auf die Zeitgenossen ausgeübt haben.

In den beiden letzten Kapiteln wird der Einfluß amerikanischer Unterhaltungsmusik auf die Tanzmusik in Deutschland angesprochen. Die USA haben - nicht zuletzt durch ihre Beteiligung am Ersten Weltkrieg noch 1917 - massiv in die politische Entwicklung des Kontinents eingegriffen, wirtschaftlich (beispielsweise mit dem Import des Jazz) Einfluß genommen und den Publikumsgeschmack in Deutschland entscheidend beeinflußt.

\section{Musik und Politik}

Mit den Unterrichtsmaterialien soll der Zusammenhang musikalischer Erscheinungen mit politisch-historischen Ereignissen einsichtig gemacht werden. Geschichte für junge Menschen attraktiv zu machen, sie als diskussionswürdige Dimension auch in den Musikunter- 
richt einzubeziehen, ist Absicht dieses Buches. An manchen Stellen, beispielsweise am Anfang, zeigt sich, wieeng dieGegenwart mit älteren Traditionen verknüpft ist. Vieleder hier ausgebreiteten Materialien stehen dem heutigen Denken näher, als man im ersten Augenblick vielleicht meint und haben ihre Aktualität keineswegs verloren.

Die hier vorgeschlagene Thematik und ihre methodische Aufbereitung läßt Schülern und Lehrern Spielraum für Eigeninitiativen. Wie in anderen Heften der Studienreihe Musik ist es auch hier möglich, Unterrichtsschwerpunkte zu setzen und Quellen, Texte oder Musikbeispiele nach Bedarf, also nach dem jeweiligen Interesse der Lerngruppe oder den Vorstellungen der Lehrerin und des Lehrers auszuwählen. Teilbereiche wie "Syncopated music", "Heil Dir im Siegerkranz«, Ausführungen über den Armeemarsch, MAHLERS »WunderhornLieder « oder die 'goldenen Zwanziger` können gesondert herausgegriffen und in anderen Unterrichtszusammenhängen verwendet werden. Die Musikbeispiele dazu sind auf einer Kassette erhältlich.

Osnabrück und Wien 1988

Sabine Schutte und Franz Niermann 


\section{Inhalt}

1. Zum Geleit: Marschmusik auf Straßen und Plätzen 9

2. „Die Musik kommt!« - Paraden und Aufmärsche in der Zeit des deutschen Kaiserreichs 11

2.1 Ein historischer Festumzug mit der "Wacht am Rhein « 12

2.1.1 Anschauungsmaterial zur »Wacht am Rhein « 13

2.1.2 Zur Funktion des Liedes 16

2.2 Oscar Straus: "Die Musik kommt!» 17

3. Preußens Gloria 29

3.1 Über den Armeemarsch 30

3.1.1 Gottfried Piefke: "Preußens Gloria» 30

3.1.2 Informationen über den Armeemarsch 34

3.2 Zur Funktion von Marschmusik 34

3.3 „Heil Dir im Siegerkranz! « 39

3.3.1 Zur Geschichte der "Kaiserhymne» 41

3.4 Nationale Hochstimmung 42

3.4.1 Johannes Brahms: »Triumphlied«, op. 5542

4. Leichte Kavallerie $\mathbf{4 4}$

4.1 Zum Beispiel: Franz von Suppé 44

4.2 Marschparodien 51

4.2.1 "Der Petersburger“ 51

4.2.2 "Auf der grünen Wiese« 54

5. Der Marsch als Ausdrucksmittel Gustav Mahler: Zwei »Wunderhorn-Lieder« 56

5.1 Gustav Mahler: "Revelge« (Franz Niermann) 56

5.1.1 Erste Übersicht: Die rein instrumentalen Teile 57

5.1.2 Anfang und Ende: Der Inhalt der ersten und letzten Strophe 57

5.1.3 Der Gesang der »Brüder« 60

5.1.4 Der Text: Gustav Mahler - Des Knaben Wunderhorn 64

5.1.5 Zusammenfassung 66

5.1.6 Nachsatz: Legende vom toten Soldaten - Bertolt Brecht (1917) 67 
5.2 Gustav Mahler: "Der Tambourg'sell« 83

5.2.1 Zum Text 83

5.2.2 Zur Melodie 84

6. Tanzschlager in Marschrhythmen 86

6.1 Der Schieber 86

6.2 Sieg der Schreittänze 94

7. Tanzzauber 96

7.1 Auf dem Ball: Zum Walzer sich dreh'n 96

7.1.1 Der erste Ball 97

7.1.2 Leo Fall: "Die Rose von Stambul« 98

7.1.3 Zur sozialen Funktion des Walzers 104

7.2 Polka 104

7.2.1 Herkunft der Polka 104

7.2.2 Tanz-Praxis: Die »Amboß-Polka« 106

8. Amerika läßt grüßen 108

8.1 "Alexander's Ragtime« 108

8.2 Vom Marsch zum Ragtime 108

8.2.1 Scott Joplin: "The Entertainer" 109

8.2.2 Der »Petersburger" als Ragtime 113

8.3 Cakewalk 113

8.3.1 Claude Debussy: „Golliwogg's Cakewalk« 114

9. Die sgoldenen zwanziger Jahre $\mathbf{1 2 0}$

9.1 Tanzfieber 120

9.2 Syncopated music 122

9.3 Der Foxtrott - Erbe von Marsch und Ragtime 127

9.3.1 Rhythmus und Tempo des Foxtrotts 132

9.4 Charleston 136

10. Anhang 141

10.1 Märsche, Lieder und Tänze 142

10.2 Notenstiche 142

10.3 Abbildungen 142

10.4 Register 143

10.5 Hörbeispiele 144 